\title{
Clinical Neuroimaging Using Arterial Spin-Labeled Perfusion Magnetic Resonance Imaging
}

\author{
Ronald L. Wolf* and John A. Detre*广 \\ Departments of *Radiology and ${ }^{\dagger}$ Neurology, University of Pennsylvania Medical Center, Philadelphia, Pennsylvania 19104
}

\begin{abstract}
Summary: The two most common methods for measuring perfusion with MRI are based on dynamic susceptibility contrast (DSC) and arterial spin labeling (ASL). Although clinical experience to date is much more extensive with DSC perfusion MRI, ASL methods offer several advantages. The primary advantages are that completely noninvasive absolute cerebral blood flow $(\mathrm{CBF})$ measurements are possible with relative insensitivity to permeability, and that multiple repeated measurements can be obtained to evaluate one or more interventions or to perform perfusion-based functional MRI. ASL perfusion and
\end{abstract}

perfusion-based functional MRI methods have been applied in many clinical settings, including acute and chronic cerebrovascular disease, CNS neoplasms, epilepsy, aging and development, neurodegenerative disorders, and neuropsychiatric diseases. Recent technical advances have improved the sensitivity of ASL perfusion MRI, and increasing use is expected in the coming years. The present review focuses on ASL perfusion MRI and applications in clinical neuroimaging. Key Words: Magnetic resonance imaging, arterial spin labeling, arterial spin tagging, perfusion, functional MRI.

\section{INTRODUCTION}

Perfusion refers to the delivery of blood flow to a tissue or organ. Brain perfusion is also termed cerebral blood flow (CBF) and is expressed in units of $\mathrm{mL} / \mathrm{g} /$ min, reflecting the volume of flow per unit brain mass per unit time. Absolute quantification of $\mathrm{CBF}$ requires a tracer that can diffuse from the vasculature into tissue. Although intravascular (nondiffusible) tracers can be used to infer CBF through the central volume principle, ${ }^{1-4}$ they do not strictly quantify CBF; however, hemodynamic measurements based on both diffusible and intravascular tracers are often referred to as perfusion imaging.

The two most common methods for measuring perfusion using ${ }^{1} \mathrm{H}$ MRI are the dynamic susceptibility contrast (DSC) approach, which detects the first passage of an intravascular contrast agent such as a gadolinium chelate, and arterial spin labeling (ASL), which uses magnetically labeled arterial blood water as a diffusible flow tracer. DSC perfusion MRI, also termed perfusionweighted imaging, is more widely applied clinically and has

Address correspondence and reprint requests to: Ronald L. Wolf, MD, PhD, Department of Radiology, Neuroradiology Section, 219 Dulles Building, 3400 Spruce St., Philadelphia, PA 19104. E-mail: Ronald.Wolf@uphs.upenn.edu. been extensively reviewed elsewhere. ${ }^{5-14}$ The present review focuses on ASL perfusion MRI, including methodological considerations and applications in basic and clinical neuroscience. Practical utility of ASL methodology has been demonstrated for several neuroimaging applications, including acute and chronic cerebrovascular disease, CNS neoplasms, epilepsy, and functional MRI (fMRI). ${ }^{9,11}$ Recent technical advances have dramatically improved the sensitivity of ASL perfusion MRI, and its use is likely to increase in the coming years.

\section{TECHNIQUE}

\section{ASL methodology}

In ASL techniques, arterial blood water is magnetically labeled using radiofrequency (RF) pulses (FIG. 1). In this it is highly analogous to CBF measurements in positron emission tomography (PET), which use water labeled with radioactive ${ }^{15} \mathrm{O}$, except that the magnetically labeled arterial water decays with $\mathrm{T} 1$ relaxation rather than a radioactive decay rate. The $\mathrm{T} 1$ relaxation time for water in blood or tissues is $\sim 1-2$ seconds, which is much more rapid than the 2-minute half-life for ${ }^{15} \mathrm{O}$. As a result, only small amounts of arterial spin-labeled water accumulate in the brain, although the temporal resolution is much faster than with $\left[{ }^{15} \mathrm{O}\right] \mathrm{H}_{2} \mathrm{O}$ PET. 


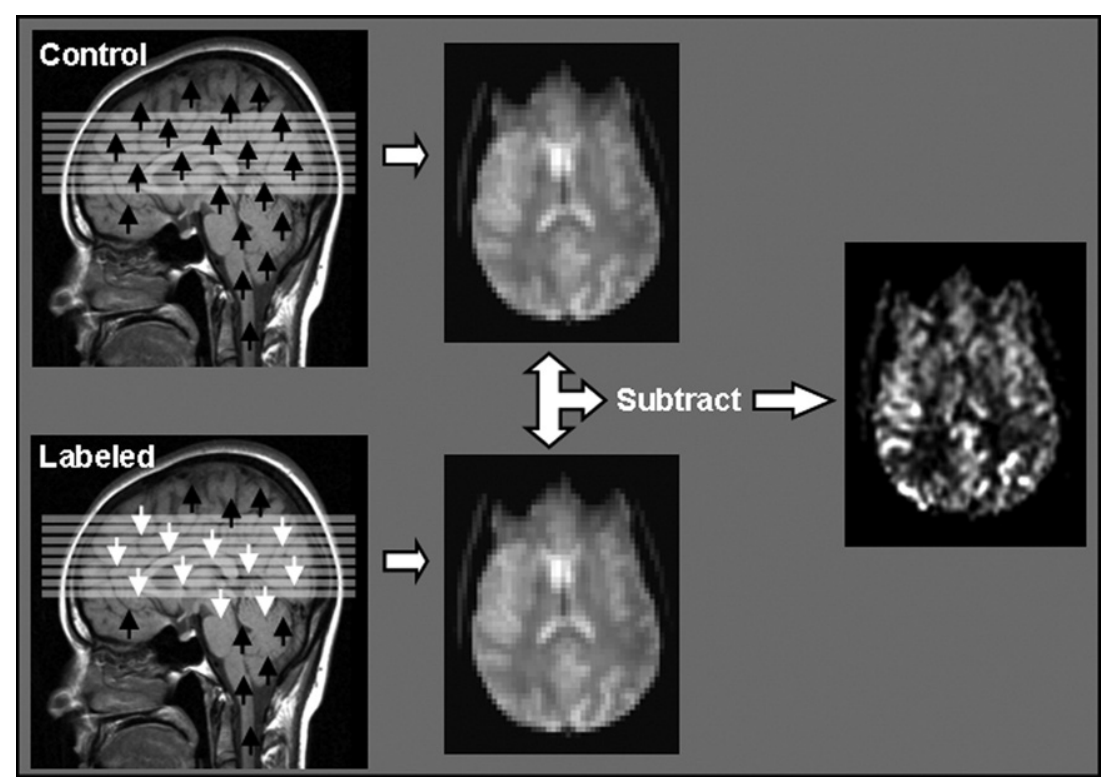

FIG. 1. Arterial spin-labeled (ASL) perfusion MRI: basic concept. Arterial blood is labeled or tagged and, after a delay, moves into the imaging plane or volume, during which time there is T1 decay of the label. Snapshot images are acquired in labeled and control conditions and subtracted, yielding a difference image with intensity proportional to cerebral blood flow (CBF).

It is critical to introduce a post-labeling delay in ASL, to allow magnetically labeled blood water to flow into the microvasculature and tissue. ${ }^{15}$ When arterial transit times are markedly prolonged, as in severe ischemia, $\mathrm{CBF}$ can be underestimated because of relaxation of the spin label. Published comparisons between ASL and $\left[{ }^{15} \mathrm{O}_{2} \mathrm{H}_{2} \mathrm{O}\right.$ PET in normal subjects demonstrate a close correlation for measured $\mathrm{CBF}$ at rest ${ }^{16}$ and with task activation, ${ }^{17}$ as would be expected.

In perfusion MRI, the effects of ASL are measured through comparison with images acquired with control labeling that does not affect the magnetization of arterial blood water, but accounts for magnetization transfer effects. During ASL image acquisition, repeated label and control images are interleaved. Perfusion contrast is obtained by pairwise subtraction of the label and control acquisitions, and absolute $\mathrm{CBF}$ in well characterized physiological units of $\mathrm{mL} / 100 \mathrm{~g} / \mathrm{min}$ can be estimated by modeling expected signal changes in the brain, primarily taking into account the tracer half-life determined by the T1 of blood (1-2 seconds). Multiple labeled-control image pairs are typically acquired and averaged for generating CBF maps. This is due to inherently low signal-tonoise ratio (SNR) of the effective signal difference (a few percent) between labeled and control images. ${ }^{5}$

During the past decade, theoretical and experimental studies have been conducted to improve the accuracy of CBF quantification using ASL by taking into account multiple parameters such as arterial transit time, ${ }^{15,18-21}$ magnetization transfer effects, ${ }^{15,22} \mathrm{~T} 1,{ }^{23,24}$ labeling efficiency, ${ }^{25-27}$ and capillary water permeability. ${ }^{28,29}$

\section{Spin labeling schemes}

There are two major approaches for ASL: continuous (CASL) and pulsed (PASL) (FIG. 2). In CASL, arterial blood water is continuously and selectively labeled as it passes through a labeling plane, typically applied at the base of the brain. ${ }^{30,31}$ Labeling of all blood water occurs at the same location, and labeling can be applied for several seconds, maximizing the effects on brain signal. In PASL, a short RF pulse is used to instantaneously invert blood and tissue, and can be applied either below the brain $^{32}$ or to the entire brain with subsequent selective inversion of the imaging slices to produce a magnetization difference between blood and brain water. ${ }^{33}$

Although CASL provides stronger perfusion contrast, ${ }^{23}$ it is more difficult to implement than PASL because of its hardware demands, and it deposits a higher level of RF power into the subject. The long labeling pulses in CASL also partially excite the imaging slices through an effect termed magnetization transfer. This has to be balanced during the control acquisitions, in order to accurately quantify $\mathrm{CBF}$, and special procedures are required to permit multislice imaging. Multislice CASL can be obtained using separate labeling and imaging coils, ${ }^{34-36}$ or by moving the control pulse to the same location as the imaging pulse and modulating it with a sinusoidal waveform so that moving blood is inverted and then reinverted, such that the net inversion is close to zero. ${ }^{22}$

PASL methods, on the other hand, require a well designed slice profile for the labeling pulse, with a sharp edge to eliminate residual signal from static tissue. Mul- 


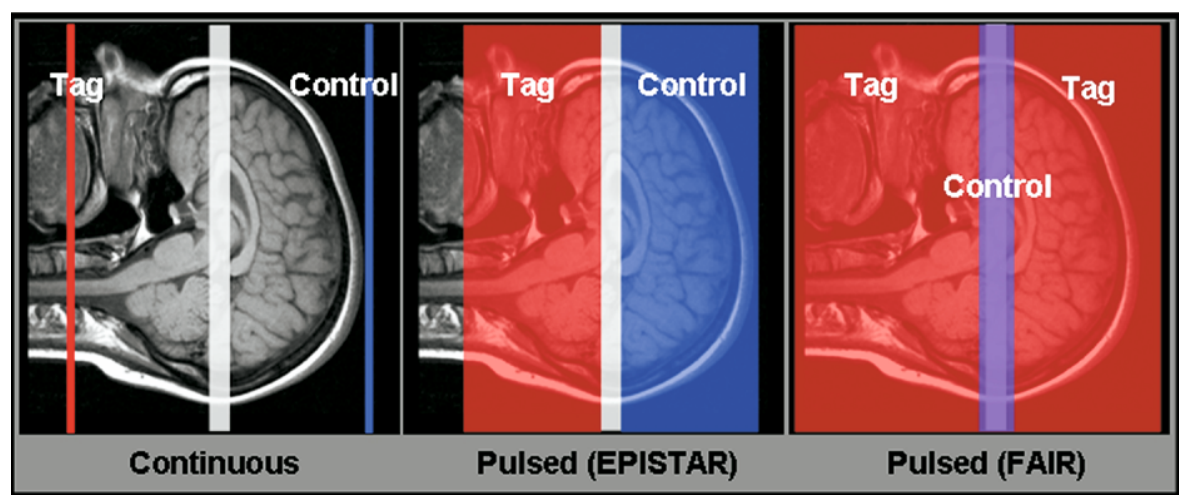

FIG. 2. Continuous $A S L$ (CASL) and pulsed ASL (PASL) methodology (single slice implementation). For CASL, arterial blood moving through the labeling plane is tagged by flow-driven adiabatic inversion. The control acquisition accounts for magnetization transfer effects but leaves arterial blood unaffected. Prototype PASL methods include EPISTAR, which uses tagging and control slab inversion, and FAIR, which uses a nonselective inversion pulse for labeling and selective inversion pulse centered over the imaging plane for a control.

tislice acquisitions have been developed also for PASL methods. ${ }^{37,38}$

\section{Improving the sensitivity of ASL}

High magnetic field strength is particularly beneficial for ASL, because not only does image SNR increase in proportion to the field, but $\mathrm{T} 1$ also lengthens, allowing more spin label to accumulate. ${ }^{23} \mathrm{~A}$ twofold signal gain is readily achievable by performing PASL/CASL at 3.0 and 4.0 $\mathrm{T}$, compared with similar methods at $1.5 \mathrm{~T}$. ${ }^{23,39}$ Higher field strengths allow improved spatiotemporal resolution, as well as longer post-labeling delay times to counteract delayed transit effects usually present in patients with cerebrovascular disease. ${ }^{40}$ As 3 T MRI scanners are increasingly available at many neuroimaging centers, high-field ASL is expected to become the standard practice, with widespread applications.

A second and complementary approach for improving SNR in ASL perfusion MRI is to use a phased array receiver. Although the signal enhancement from array coils is not homogeneous in space (higher in cortex, lower in the deeper parts of the brain), the absolute CBF maps obtained with ASL are largely unaffected by this coil sensitivity profile effect, because of several calibration steps involved in perfusion quantification. ${ }^{41}$ Phased array coils can also be optimized for parallel imaging to shorten the image acquisition time by the acceleration factor.

Although there is usually a reduction in SNR with parallel imaging, in ASL perfusion MRI, much of this SNR cost can be regained through shortened TE along with reduced distortion from susceptibility artifact. ${ }^{41} \mathrm{Ar}-$ ray receiver and parallel acquisition with body coil excitation are now becoming the standard configuration for commercial MRI scanners. CASL is particularly challenging when used with body coil excitation because of the increased RF demands on the hardware. It has recently been demonstrated, however, that CASL can be approximated by a very large number of much shorter pulses that are compatible with body coil excitation, ${ }^{42}$ an approach that has been termed pseudo-CASL.

\section{Improving ASL image acquisition}

Because the ASL effect is small, it is desirable to use an imaging sequence with high SNR. Much of the data acquired to date using ASL has applied echoplanar imaging, because of its high SNR and fast acquisition time, which reduces the potential for motion artifacts between label and control scans. Echoplanar imaging can, however, introduce distortions in regions of high static susceptibility gradients that degrade image quality. Over the past several years, fast three-dimensional (3D) sequences have been introduced for image acquisition in ASL to improve image quality. The 3D sequences provide improved SNR due to slab excitation and a prolonged image acquisition window, and image distortion arising from magnetic field inhomogeneity effects is reduced compared with routine 2D gradient-echo echoplanar imaging or spiral trajectory sequences. It is possible to acquire the whole brain volume within a single-shot RF excitation using ultrafast 3D sequences, such as combined gradient and spin-echo (GRASE). ${ }^{43}$

Another advantage of $3 \mathrm{D}$ imaging is that, because excitation of the entire imaging slab occurs at a single point in time, it facilitates the use of background suppression to reduce the static brain signal. ${ }^{44}$ Background suppression with a series of appropriately timed inversion pulses can increase the ASL effect from $\sim 1 \%$ of brain signal up to $100 \%$ of measured signal, dramatically increasing the sensitivity for detecting dynamic changes in CBF. ${ }^{45}$ Although not yet widely available, ASL imaging sequences incorporating high-field, parallel imaging, pseudo-CASL, and 3D imaging with background suppression now provide approximately a 10 -fold increase in sensitivity for imaging $\mathrm{CBF}$, compared with prior approaches (FIG. 3). 


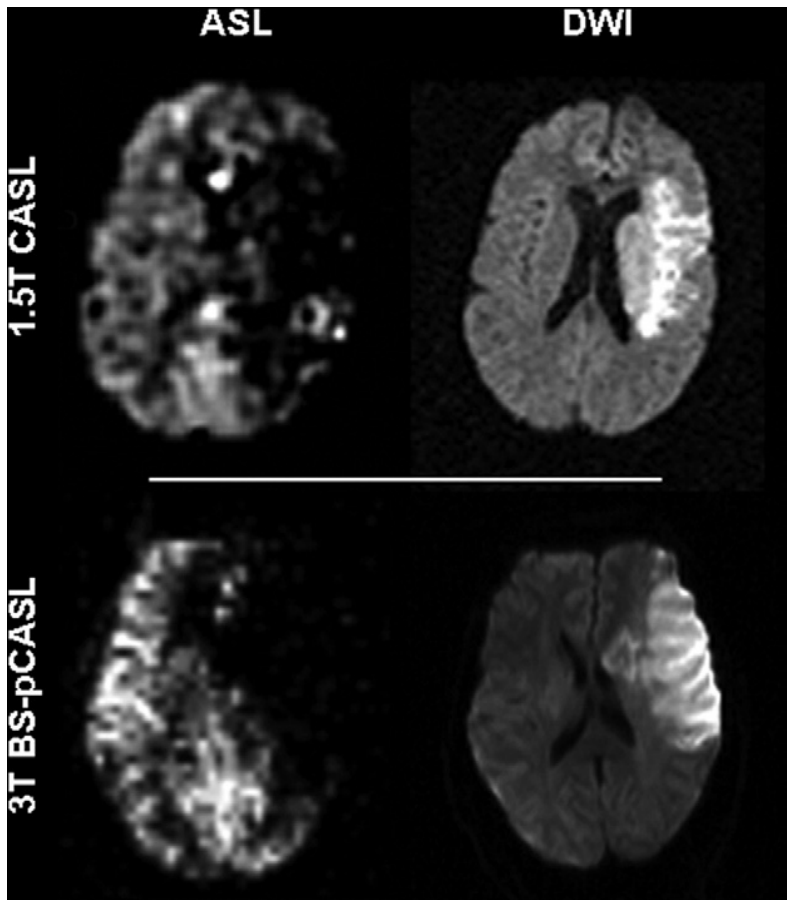

FIG. 3. Improvements in ASL methodology. Two patients with acute middle cerebral artery (MCA) infarction are shown, with selected ASL CBF maps on the left and diffusion-weighted imaging (DWI) on the right. For the first patient (top row), a continuous multislice ASL (CASL) acquisition at $1.5 \mathrm{~T}$ took 8 minutes. For the second patient (bottom), a background suppressed pseudo-CASL acquisition (BS-pCASL) at $3 \mathrm{~T}$ was completed in 32 seconds.

\section{CLINICAL APPLICATIONS}

\section{Acute and chronic cerebrovascular disease}

Siewert et al. ${ }^{46}$ first applied ASL in an acute stroke population, using the prototype single-slice PASL technique EPISTAR ${ }^{32}$ and implementing multiple inversion times. Perfusion was assessed in the region of a documented diffusion abnormality. PASL and DSC perfusion MRI results were compared qualitatively, and also semiquantitatively using relative measures compared with the contralateral normal side. They reported agreement between techniques in assessment of perfusion (hypoperfused or delayed, normal, or hyperperfused) in 17 of 21 imaging studies, and disagreement in 4. In the cases which showed disagreement, EPISTAR showed absent perfusion where DSC perfusion MRI showed a delay. Apparent absent perfusion with EPISTAR even at the longest post-inversion delay of 1500 milliseconds illustrates one of the main problems encountered in this population: long transit delay times and difficulty visualizing collateral flow due to T1-decay of label occurring too quickly.

The first study applying multislice acquisition in patients with symptomatic cerebrovascular disease, in this case using CASL, ${ }^{47}$ showed that resting perfusion deficits could be detected. Subsequently, Chalela et al. ${ }^{48}$ prospectively studied 15 acute ischemic stroke patients with CASL perfusion MRI at $1.5 \mathrm{~T}$ within 24 hours of symptom onset, correlating $\mathrm{CBF}$ values in defined vascular territories with the National Institutes of Health Stroke Scale (NIHSS) at admission and Rankin Scale (RS) at 30-day follow-up. Only patients with symptoms of supratentorial ischemia were included. Of 11 patients with perfusion deficits ( 3 of the 15 had normal perfusion and 1 showed hyperperfusion), 8 demonstrated perfusion-diffusion mismatches, with mismatches ranging from small diffusion but large perfusion abnormalities to large diffusion with small perfusion abnormalities (FIG. 3). Absolute $\mathrm{CBF}$ and interhemispheric asymmetry measures correlated with severity and outcome as determined by NIHSS and RS scores.

Delayed arterial transit effects were demonstrated in 7 of the 15 cases in the Chalela et al. ${ }^{48}$ study, and although qualitative interpretation of perfusion deficits was still possible, this illustrated a potential difficulty for absolute quantification in this setting. This was further studied in a small group of 11 patients with acute or chronic cerebrovascular disease, in whom DSC and CASL perfusion MRI methods were compared. ${ }^{49}$ Subjective and objective comparisons showed that time-to-peak (TTP) maps from DSC perfusion MRI acquisition correlated (negatively) best with CBF maps generated from CASL perfusion MRI. If those patients with major transit delays ( $n$ $=3$ ) were excluded from analysis, CBF measures generated from both DSC and CASL perfusion MRI acquisitions correlated best.

Hunsche et al. ${ }^{50}$ performed a similar comparison with DSC perfusion MRI-derived parameters and single-section flow-sensitive inversion recovery (FAIR), showing a good correlation of CBF measures from both methods in normal volunteers and patients with ischemic stroke and more modest correlation of FAIR CBF measures with DSC cerebral blood volume (CBV) and mean transit time (MTT) parameters. Yoneda et al. ${ }^{51}$ compared relative perfusion measures (asymmetry ratios of occlusive carotid disease, affected side compared with unaffected side) based on the FAIR method with 2 inversion times ( $\mathrm{TI}=800$ and 1600 milliseconds) to perfusion metrics generated with DSC perfusion MRI. They studied 11 patients, finding asymmetry ratios (AR) using FAIR at TI $=1600$ milliseconds correlated best with MTT and TTP measures from the DSC perfusion technique. Correlations were less favorable for the comparison of FAIR to relative $\mathrm{CBF}$ and especially $\mathrm{CBV}$ measures.

All of these studies encountered and discussed difficulties with transit delay, which is arguably the most substantial obstacle for quantitation and interpretation. The introduction of a postlabeling delay ${ }^{15}$ dramatically reduced transit time sensitivity, although very long delays remained a problem. Other approaches include techniques with multiple inversion times. ${ }^{7,46,51-54}$ By includ- 
ing longer delay times, up to 2400 milliseconds, Siewert et al. ${ }^{46}$ demonstrated some delayed appearance of label in the territory of interest suggestive of collateral flow, but at least at $1.5 \mathrm{~T}$ most of the label is destroyed at these longer delay times, and completely destroyed at $\sim 6 \mathrm{~s}$. Moving to a higher field strength makes this approach more feasible: the increased $\mathrm{T} 1$ allows label to persist at longer delays and improves ability to visualize potential collateral flow. $23,40,41$

As we have noted, qualitative interpretation may still be clinically useful even if not completely accurate for measuring absolute blood flow. For PASL, limiting the bolus width (e.g., QUIPSS II) improves quantification with variable transit times. Diffusion or flow-weighting gradients have also been applied to simply eliminate the intravascular signal. Velocity-selective ASL (VS-ASL) is a newer method that tags blood based on velocity and not spatial location, selectively labeling blood below a cutoff velocity and thus selecting decelerating blood. ${ }^{55-57}$ Its efficacy in patients with cerebrovascular disease has yet to be established. One can also directly measure transit time with a modification of ASL methodology. ${ }^{58}$

Many perfusion techniques using exogenous tracers allow repeated study administration of a tracer to evaluate the effect of a treatment or intervention, such as after acetazolamide administration; however, repetitions are generally limited to a baseline and post-intervention or post-treatment acquisition. ASL techniques are unique in that they allow multiple (theoretically infinite) repeated studies.

CASL perfusion MRI before and after acetazolamide challenge was described early after introduction of ASL methods. ${ }^{59}$ Ances et al. ${ }^{60}$ used multislice CASL perfusion MRI to evaluate CBF before and after carotid endarterectomy. For the anterior (but not posterior) circulation, $\mathrm{CBF}$ changes were inversely related to baseline $\mathrm{CBF}$; that is, increases after carotid endarterectomy were greater when baseline CBF was lower. They found decreased hemispheric perfusion at rest both ipsilateral (mean of $40 \mathrm{~mL} / 100 \mathrm{~g} / \mathrm{min}$ ) and contralateral (mean of $43 \mathrm{~mL} / 100 \mathrm{~g} / \mathrm{min}$ ) to side of carotid endarterectomy, with significant increases in absolute global CBF in patients with baseline decreased CBF $(<50 \mathrm{~mL} / 100 \mathrm{~g} / \mathrm{min})$ prior to carotid endarterectomy.

The ability to reliably measure absolute perfusion instead of relying on relative measures is clearly advantageous, not only because it is possible to directly measure tissue at risk, but also because relative measures are no longer reliable when vascular disease is bilateral or when cardiac output decreases. Multiple studies have evaluated reliability and reproducibility of ASL perfusion measurements, ${ }^{16,61-65}$ making cross-sectional and longitudinal studies increasingly powerful and simplifying comparisons with other measures of function and performance.
For example, using a CASL perfusion method at $1.5 \mathrm{~T}$, Floyd et al. ${ }^{61}$ reported a within-subject coefficient of variation $(\mathrm{CV})$ of $5.8 \%$ for global and $13 \%$ for territorial CBF measurements with repeated studies within 1 hour. Global and territorial within-subject CVs were $13 \%$ and $14 \%$, respectively, when studies were repeated in 1 week. Using a PASL perfusion method at $1.5 \mathrm{~T}$, Jahng et al. ${ }^{62}$ reported a fairly high reliability (with intraclass correlation coefficient of 0.75 ) and reproducibility (with CV less than $8.5 \%$ ) when subjects were studied twice within 2 hours. They also noted that random noise related to the inherently low SNR of the ASL perfusion technique was the primary factor limiting reliability and reproducibility (not within-subject variability, at least not for repeated measures over a short term). Higher fieldstrength imaging with improved SNR methods would thus be expected to improve reliability.

The utility of reliable absolute perfusion measures is illustrated in a case study describing a patient with moyamoya syndrome studied before and after superficial temporal to middle cerebral artery bypass. ${ }^{66}$ Improved absolute hemispheric CBF values were reported, increasing from 20 and $27 \mathrm{~mL} / 100 \mathrm{~g} / \mathrm{min}$ on right and left, respectively, to a corresponding 53 and $56 \mathrm{~mL} / 100 \mathrm{~g} / \mathrm{min}$. Neuropsychological testing showed improvements in verbal learning and memory metrics before and after surgery. Of additional interest are studies addressing relationships of cerebral atrophy and blood flow to systemic factors related to atherosclerosis and other cardiovascular risk factors, as well as stenotic-occlusive disease. $^{67,68}$

For perfusion MRI, unique to ASL methodology is the ability to separately label individual vascular territories. Implementations have included use of a separate labeling coil,${ }^{35}$ tilted labeling planes or slabs, ${ }^{69-71}$ and strategies for selective labeling of individual arteries. ${ }^{72,73}$ Hendrikse et al. ${ }^{74}$ studied the hemodynamic effects in the brain of extracranial to intracranial bypass in seven patients with occluded carotid arteries, finding that the flow territory of the bypass was smaller than that of the contralateral internal carotid artery, but that the absolute $\mathrm{CBF}$ in the hemisphere supplied by the bypass was not substantially different from that on the contralateral side or in control subjects. Jones et al., ${ }^{70}$ using a unilateral labeling scheme to study the effects of carotid endarterectomy on CBF, extended the work of Ances et al. ${ }^{60}$ by separating the contributions of the operated and nonoperated side. Before surgery, they found decreased CBF in the middle cerebral artery (MCA) territory supplied by the more stenotic (ipsilateral) carotid artery, although there was no difference in hemispheric blood flow because the contribution from the less stenotic (contralateral) side to the ipsilateral MCA was increased (FIG. 4). Flow to the MCA territory on the side of surgery supplied by the ipsilateral carotid artery increased the most 


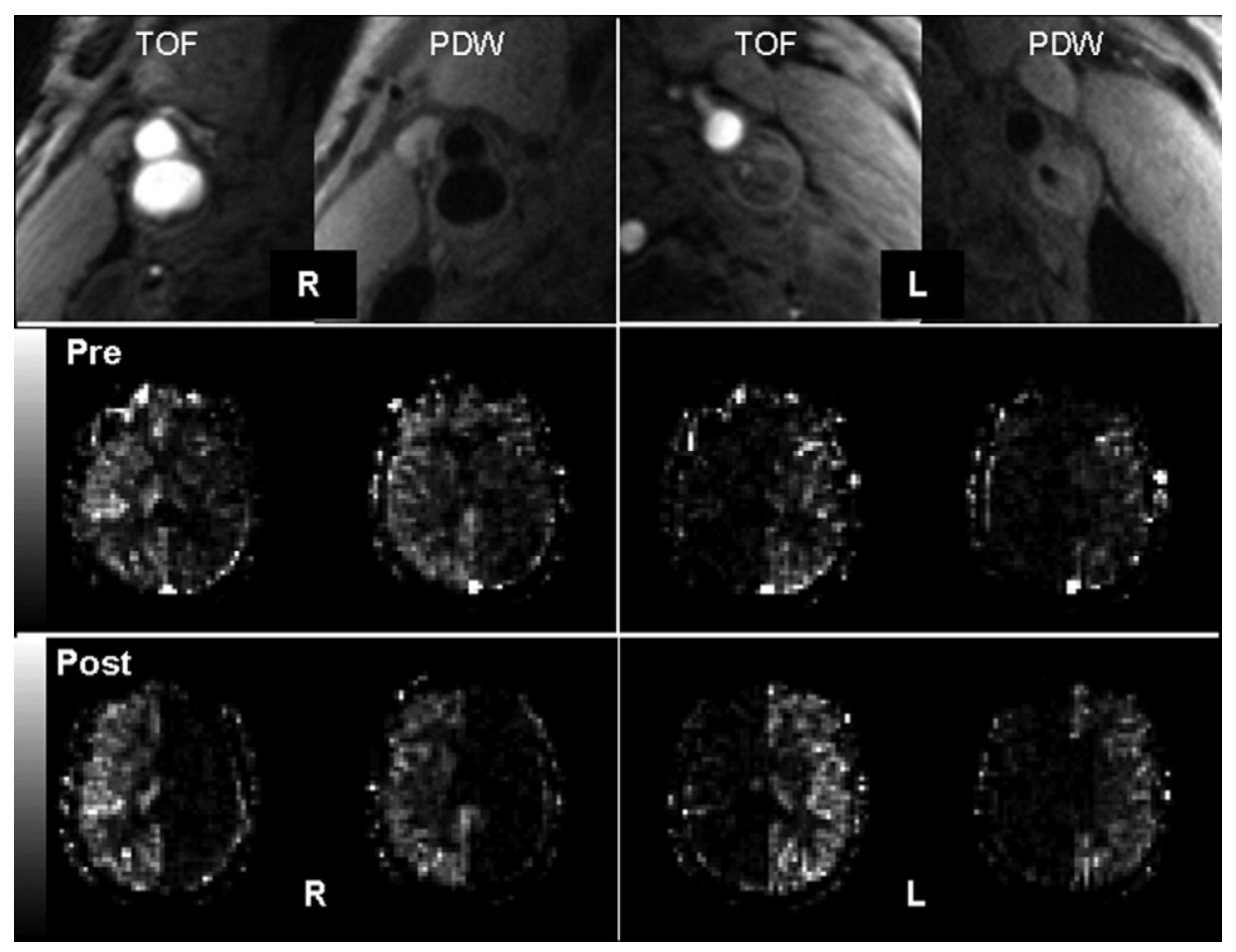

FIG. 4. Selective (unilateral) labeling strategy for determining relative contributions of cervicocranial vessels to vascular territories in the brain. (Top) Axial bright-blood time of flight (TOF) images and black-blood proton density-weighted (PDW) images just above bifurcation show high grade stenosis on left (L). (Middle) CBF maps with right and left sided labeling pre-endarterectomy on left show much of the left MCA territory CBF is supplied from the right side. (Bottom) Post-endarterectomy, hemispheric CBF is more symmetrically supplied according to right and left labeling.

for the patients with the lowest CBF contribution from the ipsilateral carotid preoperatively, and the contribution from the contralateral carotid artery decreased accordingly. Because blood flow territories can vary between individuals, these techniques obviously need to take into account collateral pathways through the circle of Willis and elsewhere. ${ }^{75}$

\section{Vascular malformations}

Transit effect also becomes an issue in the setting of vascular malformations, in particular arteriovenous malformations (AVMs). For blood moving through an AVM, abnormal shortening of transit time is present and blood labeled using ASL methods no longer functions as a diffusible tracer but as an intravascular tracer. This effect was originally implemented as a means for generating MR angiograms. ${ }^{76-78}$ In this application, ASL difference images display AVMs and draining vessels as high-contrast structures with an angiographic appearance, whereas the rest of the brain shows perfusion effects where CBF can still be calculated (FIG. 5). This effect was exploited in preliminary work investigating the feasibility of using ASL perfusion MRI to detect AVMs, quantify the resulting arteriovenous shunting, and evaluate effects on adjacent and distant regional $\mathrm{CBF}^{79}$

\section{CNS neoplasms}

Perfusion studies using MRI in this setting are more commonly implemented with DSC perfusion methods, typically focusing on the calculated parameter relative CBV (rCBV). An advantage of ASL methodology in this setting is that use of a diffusible tracer (labeled arterial water) renders ASL relatively insensitive to permeability. ${ }^{80}$ The completely noninvasive nature of these methods is also very convenient; for example, intravenous access with a large-bore cannula is not necessarily trivial for pediatric age groups and chemotherapy patients. Disadvantages include the low SNR, relative to DSC perfusion MRI methods. Also, although the parameter CBF seems to have diagnostic significance similar to $\mathrm{CBV}$, calculation of $\mathrm{CBV}$ or transit time, though not impossible, is not as efficiently accomplished with ASL techniques. ${ }^{52,54,58}$

Permeability has also been shown to be of interest in evaluating CNS neoplasms and treatment effects. A preliminary report suggests that a modification of the ASL technique may allow access to this parameter as well. ${ }^{81}$ Absolute measures of $\mathrm{CBF}$ in brain tumor populations may not be as critical as in assessment of cerebrovascular disease, given heterogeneity in populations because of age and histopathology, and relative measures comparing 

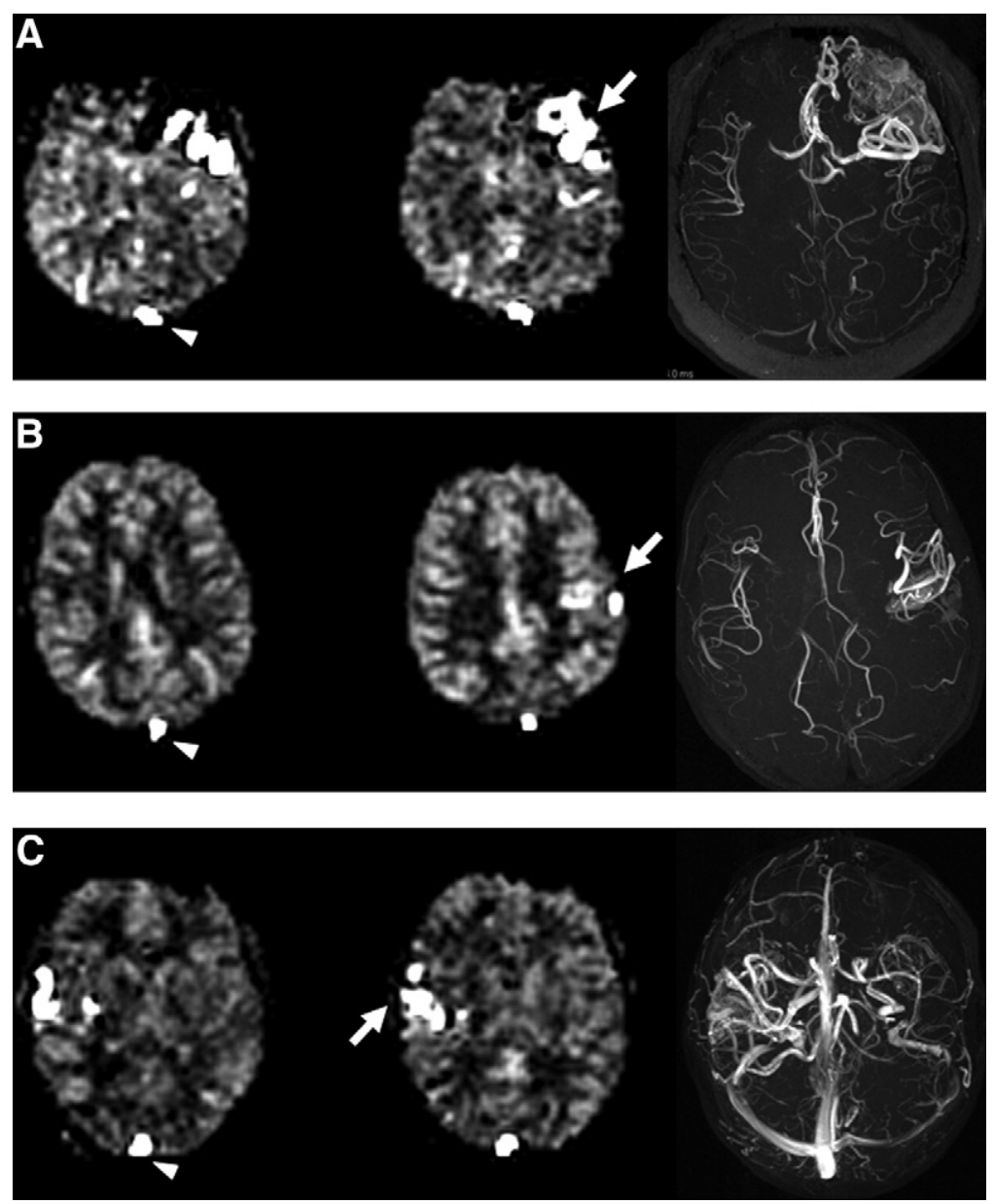

FIG. 5. Evaluation of arteriovenous malformations (AVMs) with CASL perfusion MRI. (A-C) Each row corresponds to a different patient, with 2 of 12 CASL imaging locations on the left and slab maximum intensity projections from 3D time of flight magnetic resonance angiogram (MRA) through the AVM on the right. AVM nidus and draining vessels (arrows) are clearly seen on CASL CBF maps, as is labeling in cortical veins and sagittal sinus (arrowhead) indicating rapid transit or arteriovenous (AV) shunting.

to global CBF or white matter will likely be of greater practical use. ${ }^{56,82}$

Gaa et al. ${ }^{83}$ first reported the use of ASL in brain tumors, applying EPISTAR. Warmuth et al. ${ }^{82}$ reported the first comparison of ASL CBF measures with perfusion parameters generated from DSC perfusion MRI for the evaluation of both primary and secondary brain neoplasms before and after treatment. They used a PASL approach implemented at $1.5 \mathrm{~T}$. Coverage was limited to three imaging sections, but they were able to show that both ASL and DSC perfusion MRI approaches allowed a distinction between high-grade and low-grade gliomas, with higher tumor blood flow (TBF) and blood volume in high-grade gliomas (and metastases). In general, mean TBF was higher than mean CBF in high-grade gliomas and lower than mean CBF in low-grade gliomas. Wolf et al., ${ }^{80}$ used a multislice (12 imaging sections) CASL technique implemented on a $3 \mathrm{~T}$ scanner, also found that high-grade gliomas could be distinguished from lowgrade gliomas preoperatively, with some exceptions when oligodendroglial components were present. Us- ing measures of maximum blood in the tumor normalized to global CBF $\left(\mathrm{nTBF}_{\max }\right.$ ), in general high-grade gliomas showed $\mathrm{nTBF}_{\max }>1.3$ and low-grade gliomas showed $\mathrm{nTBF}_{\max }<1.3$ (FIG. 6). Kimura et al. ${ }^{84}$ applied CASL perfusion CBF measures to evaluation of meningiomas and compared them to DSC perfusion MRI parameters, finding comparable measurements of $\mathrm{CBF}$ with the highest $\mathrm{CBF}$ values in the angiomatous histologic subtype.

A recent study evaluated PASL perfusion MRI (Q2TIPS as well as ITS-FAIR) in conjunction with magnetic resonance spectroscopic imaging (MRSI), DSC perfusion MRI, and dynamic contrast-enhanced MRI for grading CNS neoplasms, ${ }^{85}$ comparing imaging to histologic grade and features such as vessel density and proliferation index. They found that both DSC and ASL perfusion MRI (Q2TIPS performing slightly better than ITS-FAIR) were comparable for grading gliomas and better for discriminating glioblastoma from lymphoma, metastatic disease (where peritumoral signal T2-hyperintense regions were used to distinguish glioblastoma 

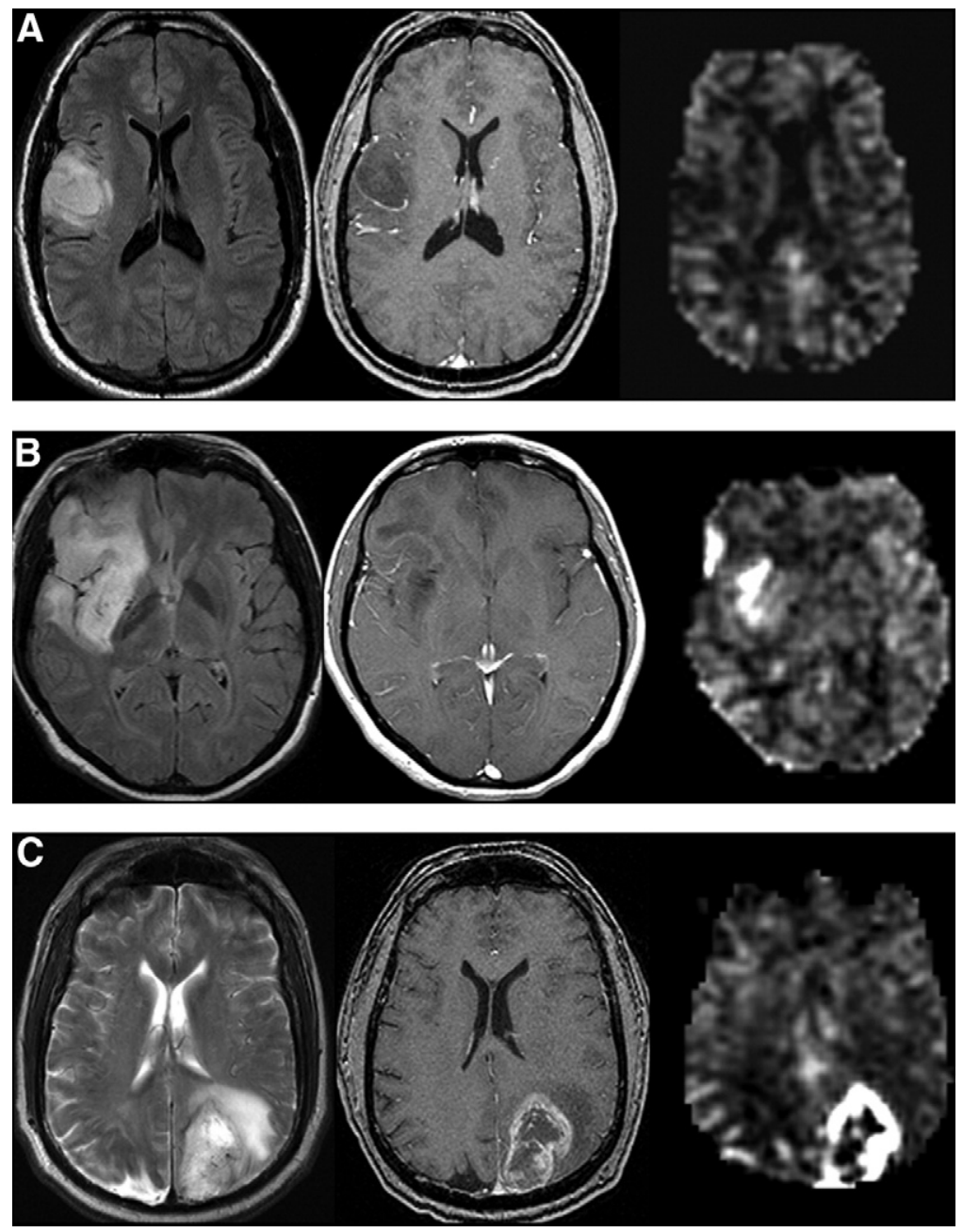

FIG. 6. CASL perfusion MRI for evaluation of gliomas. (A) Oligodendroglioma (WHO grade II). (B) Anaplastic oligoastrocytoma (grade III). (C) Glioblastoma (grade IV).

from metastasis), and lower glioma grades. Specificity was not as good for distinguishing glioblastoma from grade 3 gliomas, however, or for distinguishing grade 3 from grade 2 gliomas. Law et al. ${ }^{86}$ also found that rCBV measures generated using DSC perfusion MRI data were better for grading gliomas, with or without MRSI.

These studies underscore a well known difficulty with grading CNS gliomas using perfusion, spectroscopy, or other imaging metrics, namely, that the gold standard for comparison of histopathologic grading is also imperfect. Perhaps more relevant are comparisons with outcome measures. Weber et al. ${ }^{87}$ used PASL and DSC methods to study perfusion in brain metastases. They found that relative TBF measures (generated using both approaches) in metastases treated with stereotactic radiosurgery at 6-week follow-up were predictive of therapeutic outcome in that decreased TBF predicted response, but that pretreatment TBF measures were not predictive. This is concordant with prior studies using DSC perfusion MRI metrics. ${ }^{88-90}$ Formal studies evalu- ating ASL methods in distinguishing treatment-related necrosis from tumor recurrence are not yet available.

\section{Epilepsy}

In adults, complex partial seizures, typically arising in the temporal lobe, are the most common etiology of medically intractable seizures. These patients undergo extensive testing, including any or all of MRI, EEG, PET, fMRI, and intracarotid amobarbital (Amytal) testing, and in some cases also ictal single-photon emission computed tomography (SPECT) and proton MR spectroscopy. Interictal ${ }^{18}[\mathrm{~F}]$ fluorodeoxyglucose PET $\left({ }^{18}\right.$ FDG PET) lateralizes the side of seizure origin in temporal lobe epilepsy, based on measures of hypometabolism on the affected side. Initial comparisons showed that hypometabolism measured with ${ }^{18} \mathrm{FDG}$ PET was better in lateralizing seizures compared with interictal blood flow measurements using $\left[{ }^{15} \mathrm{O}\right] \mathrm{H}_{2} \mathrm{O}$ PET due to flow-metabolism uncoupling. ${ }^{91-93}$ Both ASL and DSC perfusion MRI methods have been applied to sei- 
zure lateralization in temporal lobe epilepsy, ${ }^{94-96}$ but in general neither method has been extensively studied in this setting.

Using DSC perfusion MRI and analyzing $\mathrm{rCBV}$ in mesial temporal lobe structures, Wu et al. ${ }^{96}$ demonstrated decreased rCBV interictally and increased ictal rCBV on the side of seizures. The lateralization correlated with ${ }^{18}$ FDG PET predictions. Similar findings were reported based on interictal CBF measures generated by multislice CASL perfusion MRI. ${ }^{95}$ In this study, temporal lobe epilepsy patients showed greater asymmetry in mesial temporal CBF compared with controls, and lateralization of seizures correlated with ${ }^{18}$ FDG PET hypometabolism, hippocampal volumes, and clinical evaluation. Global CBF measurements were also decreased in seizure patients compared with controls. Liu et al. ${ }^{97}$ applied a single-slice PASL perfusion technique (FAIRHASTE) in patients with temporal lobe epilepsy, also demonstrating interictal hypoperfusion on the side of seizures which correlated with $\mathrm{CBF}$ measures generated with $\left[{ }^{15} \mathrm{O}\right] \mathrm{H}_{2} \mathrm{O}$ PET; however, the single-slice acquisition used in this study may have magnified errors related to anatomical asymmetry. Corrections for underlying atrophy should be possible, and at least one study showed evidence of dissociation between volume and function. ${ }^{95}$

Perfusion imaging in temporal lobe epilepsy poses several other unique problems. First, the potential for unilateral or bilateral temporal lobe atrophy notwithstanding, the mesial temporal lobe structures have a relatively small volume at baseline. With the small measured signal change of a few percent in ASL, the potential for error is obvious, although it is at least in part accounted for with multiple averages. Both ASL and DSC perfusion MRI methods may suffer from susceptibility artifacts in mesial temporal regions because of the proximity of the skull base and air-containing structures. Liu et al. ${ }^{97}$ accounted for this to a degree with the FAIRHASTE technique, which minimizes susceptibility effects. Several other strategies for minimizing susceptibility artifact have been described, including GRASE, 3D fast spin echo-based (RARE) methods ${ }^{5,43,98,99}$ and using spin-echo instead of gradient-echo echoplanar imaging. ${ }^{100}$ These issues also apply to other clinical applications, especially evaluation of degenerative disorders such as Alzheimer's disease (AD) and perfusion fMRI (e.g., for memory).

Improvements in hardware and software allow increasingly sophisticated approaches to perfusion MRI in epilepsy and other disorders. MRI-compatible EEG systems have enabled improved colocalization of EEG and MRI metrics in studies of epilepsy. For example, in a recent study of patients with idiopathic generalized epilepsy, Stefanovic et al. ${ }^{101}$ studied coupling of perfusion measured with a PASL perfusion method (QUIPSS II) and metabolism (oxygen consumption, or $\mathrm{CMRO}_{2}$ ) in rela- tion to interictal epileptiform discharges and the blood oxygen level dependent (BOLD) response to a motor task in ipsilateral and contralateral primary motor cortices. The motor task produced contralateral excitation and ipsilateral inhibition. They showed coupling between $\mathrm{CBF}$ and $\mathrm{CMRO}_{2}$ in epilepsy patients for motor tasks and for interictal epileptiform discharge-induced changes, but a decreased ratio of the magnitude of blood flow changes between contralateral and ipsilateral regions in patients compared with controls.

\section{Development, aging, and degenerative diseases}

The normal development and aging process must be taken into account when performing and interpreting perfusion studies of the brain. For ASL, evaluation of the pediatric age group provides certain advantages compared with the adult: 1) increased equilibrium magnetization and prolonged T1/T2 values lead to increased tracer concentration and longer tracer half-life; 2) shorter transit times in children also lead to longer effective tracer half-life; and 3) baseline-increased CBF in children compared with adults. ${ }^{102}$ In adults, SNR of ASL signal decreases with age, as does global CBF. ${ }^{80,103}$ Parkes et al. ${ }^{63}$ found a decrease in gray matter perfusion by $0.45 \%$ per year. Floyd et al. ${ }^{104}$ studied the relative contributions of age, anemia and CASL perfusion MRI measures of $\mathrm{CBF}$ in patients undergoing cardiopulmonary bypass. They found that CBF increases dramatically after cardiac surgery with cardiopulmonary bypass, and that anemia was an important factor in this change. They also found that the magnitude of the CBF change with anemia increased with age.

In the clinical setting, imaging is commonly implemented in evaluating patients with delirium or memory loss. Conventional imaging is often nonspecific, but newer imaging methods and postprocessing techniques are becoming important in studying these patients in the research setting, and will, one can hope, translate to routine clinical use. ${ }^{105}$ MRI techniques comprise a large share of such techniques, and include anatomic or volumetric imaging, BOLD fMRI, ASL perfusion MRI and fMRI, diffusion tensor imaging, and perhaps blood-brain barrier imaging or, ultimately, direct amyloid plaque imaging. Application of perfusion methodology may be helpful in detection of delirium etiology and for predicting elevated risk of developing delirium or cognitive decline. ${ }^{105}$

One of the first studies applying ASL methodology in neurodegenerative illness was reported by Sandson et al. ${ }^{106}$ They used EPISTAR in a comparison of patients with Alzheimer's disease (AD) to age-matched controls. They found significantly lower ASL signal changes in parietal-occipital and temporal-occipital regions in $\mathrm{AD}$ patients, and a correlation between parietal-occipital per- 
fusion decreases with clinical disease severity as measured by the Blessed Dementia Scale.

Alsop et al. ${ }^{107}$ used a multislice (16 imaging sections) CASL perfusion MRI method to study 18 patients with probable $\mathrm{AD}$, comparing to 11 matched control subjects and performing a voxel-wise analysis in a standard anatomical space. They found significant decreases in CBF in temporal, parietal, frontal, and posterior cingulated cortices in AD subjects compared with controls. They also found significant correlation with patterns of decreased regional $\mathrm{CBF}$ and clinical severity of disease (measured by the Mini-Mental State Examination); specifically, parietal and posterior cingulated blood flow (but not temporal) decreased as severity increased. Limitations with regard to evaluation of changes in temporal lobe regions are again noted, but improvements in hardware and software are becoming available to address this, at least in part.

A more recent study ${ }^{108}$ used a PASL technique in AD and mild cognitive impairment (MCI) patients compared with controls, performing group comparisons with and without corrections for tissue content in perfusion voxels (partial volume effects), and also with and without corrections for global perfusion. After correction for partial volume and global perfusion effects, the AD group showed decreased perfusion in right inferior parietal lobe and bilateral middle frontal gyri compared with controls. Differences between the MCI group and controls were evident in the right parietal lobe, but with lower statistical threshold. Differences between AD and MCI groups (after correction for partial volume effects and global perfusion) were detected at the lower statistical threshold in bilateral inferior parietal lobes, posterior cingulate gyri and precuneus.

Another study applied PASL perfusion MRI in a group comparison study of patients with frontotemporal dementia (FTD) or AD versus normal controls. ${ }^{109}$ Also accounting for partial volume effects, they found a pattern of decreased right frontal lobe perfusion in the FTD group compared with the control group, which correlated with cognitive measures of judgment and problem solving. Combining perfusion results with gray matter atrophy from structural images improved discrimination of FTD from AD and from normal aging. Perfusion was maintained in parietal regions in the FTD group, as compared to the $\mathrm{AD}$ group.

Fong et al. ${ }^{110}$ reported a preliminary study with group comparisons of patients with mild probable AD or mild probable diffuse Lewy body disease (DLBD) with normal controls based on CBF measured using a 3D fast spin echo CASL approach. They found significant decreases in CBF for DLBD patients in roughly the same distribution but with greater degree of hypoperfusion compared with the $\mathrm{AD}$ group; however, the DLBD group showed less impairment on cognitive testing compared with the AD patients. Although discrimination between DLBD and AD groups would likely be limited because of similar regional patterns of hypoperfusion, they postulated that degree of hypoperfusion might be associated with decreased cholinergic function, because this is more severe in DLBD than AD.

\section{Pediatric studies}

Because it is completely noninvasive, ASL perfusion is very attractive for pediatric and neonatal applications. ${ }^{102}$ Licht et al. ${ }^{111}$ studied 25 neonates with severe congenital heart disease with PASL perfusion MRI and found that periventricular leukomalacia was associated with decreased baseline CBF and decreased cerebrovascular reserve as measured by $\mathrm{CO}_{2}$ reactivity. Several small sample populations of pediatric patients with sickle cell disease have also been studied using ASL perfusion methodology. ${ }^{112,113}$

Strouse et al. ${ }^{113}$ evaluated 24 children with sickle cell disease with CASL perfusion MRI and compared results to neurocognitive studies as well as transcranial Doppler measurements. They found an inverse relationship between performance intelligence quotient and CBF, and concluded that children with cognitive impairment could potentially be identified with CASL perfusion MRI prior to detection with transcranial Doppler or by identifying structural damage on conventional MRI. Elevated baseline CBF was also reported, concordant with prior reports based on PET or xenon computed tomography (CT) perfusion methods. This has been attributed at least in part to low hematocrit.

Oguz et al. ${ }^{112}$ were also able to detect perfusion deficits without evident abnormality in large vessels or substantial parenchymal abnormalities on conventional magnetic resonance angiography (MRA) and MRI studies. Other potential applications in the pediatric age group such as epilepsy, CNS neoplasms, and fMRI have not been extensively studied to date, but these have been discussed for adults in other sections of the present review and have similar implications for children.

\section{Perfusion-based fMRI}

Although ASL perfusion fMRI has received much less attention than BOLD fMRI, it offers a variety of advantages. ASL perfusion MRI can noninvasively quantify $\mathrm{CBF}$ at rest and with task activation, which is important for clinical populations and pharmacological or physiological interventions that can affect resting CBF. Because regional $\mathrm{CBF}$ is coupled to regional metabolism even in the absence of a specific task, resting CBF measured by ASL also provides a task-independent measure of brain function, analogous to ${ }^{18}$ FDG PET. Correlation of regional hypoperfusion with behavioral performance outside of the MRI scanner provides a means of functional brain mapping that does not depend on task performance during the scan, which is particularly advanta- 


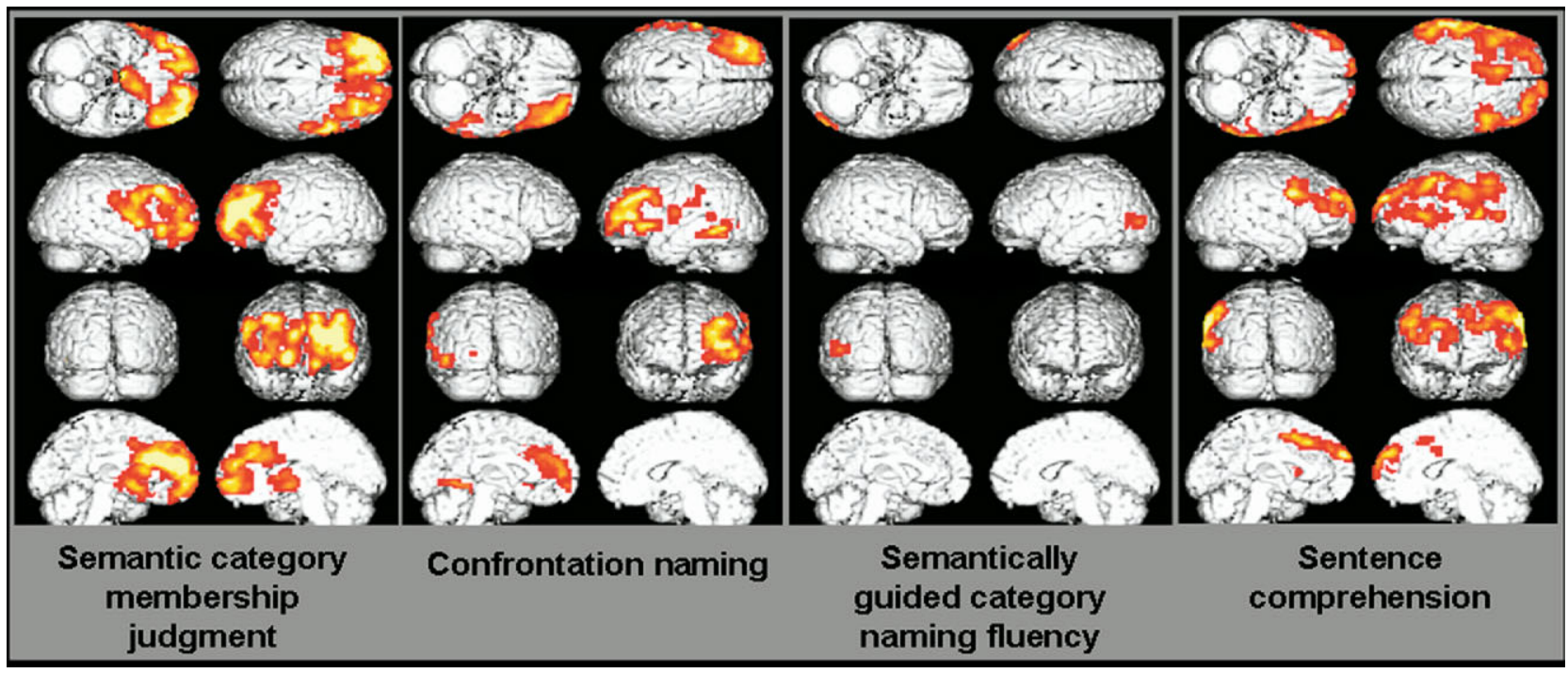

FIG. 7. Group correlations of resting CBF with language testing in patients with frontotemporal dementia $(n=16)$. Differing patterns of hypoperfusion are associated with performance deficits on varying cognitive tasks. Courtesy of D. Alsop and M. Grossman.

geous in populations with severe task performance deficits. Figure 7 shows an example of functional maps generated by correlating resting CBF with language deficits in a cohort of patients with FTD.

The frequency sensitivity of BOLD fMRI is limited by both the hemodynamic response time and low-frequency drift effects. ${ }^{114}$ Because ASL perfusion data are acquired by alternating labeled and control images, low-frequency drift effects are eliminated, ${ }^{115}$ and ASL perfusion MRI can be used to detect functional changes over minutes, hours, or even days. ${ }^{116}$ Because ASL perfusion MRI measures a purely biological parameter $(\mathrm{CBF}$, in $\mathrm{mL} / \mathrm{g} /$ $\mathrm{min}$ ), it also provides a stable and reproducible measure of regional brain function that should be independent of scanner effects. These properties should make ASL perfusion fMRI particularly useful for longitudinal or multisite studies.

Basic and cognitive neuroscience applications of ASL to date have included the use of ASL to examine brain development ${ }^{103}$ and motor learning, ${ }^{117}$ to correlate personality factors with regional brain function, ${ }^{118}$ to study brain endophenotypes associates with genotype variations, ${ }^{119}$ and to examine behavioral states such as attention, ${ }^{120}$ natural vision, ${ }^{121}$ and psychological stress. ${ }^{122}$

\section{CONCLUSION}

ASL perfusion MRI provides a robust method for quantifying CBF using MRI. The feasibility of ASL perfusion and perfusion-based fMRI methods has clearly been demonstrated for several clinical neuroimaging applications, including acute and chronic cerebrovascular disease, CNS neoplasms, epilepsy, aging and development, neurodegenerative disorders, and neuropsychiatric disease. Recent technical developments have dramati- cally improved the sensitivity of ASL perfusion MRI, paving the way for increased use of ASL perfusion MRI in clinical neuroscience and clinical practice as greater experience with this approach accrues.

Acknowledgments: This study was supported by the following grants: NIH K23 NS43381 (R.L.W.); NIH RR002305, HD050836 (J.A.D.).

\section{REFERENCES}

1. Meier P, Zierler KL. On the theory of the indicator-dilution method for measurement of blood flow and volume. J Appl Physiol 1954;6:731-744.

2. Stewart GN. Researches on the circulation time in organs and on the influences which affect it: parts I-III. J Physiol 1893;15:1-89.

3. Axel L. Cerebral blood flow determination by rapid-sequence computed tomography: theoretical analysis. Radiology 1980;137: 679-686.

4. Zierler KL. Theoretical basis of indicator-dilution methods for measuring flow and volume. Circ Res 1962;10:393-407.

5. Atlas SW, editors. Magnetic resonance imaging of the brain and spine. Philadelphia: Lippincott Williams \& Wilkins, 2002:215238.

6. Bammer R, Skare S, Newbould R, et al. Foundations of advanced magnetic resonance imaging. NeuroRx 2005;2:167-196.

7. Barbier EL, Lamalle L, Decorps M. Methodology of brain perfusion imaging. J Magn Reson Imaging 2001;13:496-520.

8. Calamante F, Thomas DL, Pell GS, Wiersma J, Turner R. Measuring cerebral blood flow using magnetic resonance imaging techniques. J Cereb Blood Flow Metab 1999;19:701-735.

9. Latchaw RE, Yonas H, Hunter GJ, et al.; Council on Cardiovascular Radiology of the American Heart Association. Guidelines and recommendations for perfusion imaging in cerebral ischemia: A scientific statement for healthcare professionals by the writing group on perfusion imaging, from the Council on Cardiovascular Radiology of the American Heart Association. Stroke 2003;34: 1084-1104.

10. Ostergaard L. Principles of cerebral perfusion imaging by bolus tracking. J Magn Reson Imaging 2005;22:710-717.

11. Wintermark M, Sesay M, Barbier E, et al. Comparative overview of brain perfusion imaging techniques. Stroke 2005;36:e83-e99.

12. Cha S. Update on brain tumor imaging. Curr Neurol Neurosci Rep 2005;5:169-177. 
13. Cha S. Dynamic susceptibility-weighted contrast-enhanced perfusion MR imaging in pediatric patients. Neuroimaging Clin $\mathrm{N}$ Am 2006;16:137-147, ix.

14. Lev MH, Rosen BR. Clinical applications of intracranial perfusion MR imaging. Neuroimaging Clin N Am 1999;9:309-331.

15. Alsop DC, Detre JA. Reduced transit-time sensitivity in noninvasive magnetic resonance imaging of human cerebral blood flow. J Cereb Blood Flow Metab 1996;16:1236-1249.

16. Ye FQ, Berman KF, Ellmore $\mathrm{T}$, et al. $\mathrm{H}_{2} 15 \mathrm{O}$ PET validation of steady-state arterial spin tagging cerebral blood flow measurements in humans. Magn Reson Med 2000;44:450-456.

17. Feng CM, Narayana S, Lancaster JL, et al. CBF changes during brain activation: fMRI vs. PET. Neuroimage 2004;22:443-446.

18. Gonzalez-At JB, Alsop DC, Detre JA. Cerebral perfusion and arterial transit time changes during task activation determined with continuous arterial spin labeling. Magn Reson Med 2000; 43:739-746.

19. Wong EC, Buxton RB, Frank LR. Quantitative imaging of perfusion using a single subtraction (QUIPSS and QUIPSS II). Magn Reson Med 1998;39:702-708.

20. Yang Y, Engelien W, Xu S, Gu H, Silbersweig DA, Stern E. Transit time, trailing time, and cerebral blood flow during brain activation: measurement using multislice, pulsed spin-labeling perfusion imaging. Magn Reson Med 2000;44:680-685.

21. Ye FQ, Mattay VS, Jezzard P, Frank JA, Weinberger DR, McLaughlin AC. Correction for vascular artifacts in cerebral blood flow values measured by using arterial spin tagging techniques. Magn Reson Med 1997;37:226-235.

22. Alsop D, Detre J. Multisection cerebral blood flow MR imaging with continuous arterial spin labeling. Radiology 1998;208:410416.

23. Wang J, Alsop DC, Li L, et al. Comparison of quantitative perfusion imaging using arterial spin labeling at 1.5 and 4.0 tesla. Magn Reson Med 2002;48:242-254.

24. Wong EC, Buxton RB, Frank LR. A theoretical and experimental comparison of continuous and pulsed arterial spin labeling techniques for quantitative perfusion imaging. Magn Reson Med 1998;40:348-355.

25. Maccotta L, Detre JA, Alsop DC. The efficiency of adiabatic inversion for perfusion imaging by arterial spin labeling. NMR Biomed 1997;10:216-221.

26. Roberts DA, Detre JA, Bolinger L, Insko EK, Leigh JS Jr. Quantitative magnetic resonance imaging of human brain perfusion at $1.5 \mathrm{~T}$ using steady-state inversion of arterial water. Proc Natl Acad Sci U S A 1994;91:33-37.

27. Utting JF, Thomas DL, Gadian DG, Ordidge RJ. Velocity-driven adiabatic fast passage for arterial spin labeling: results from a computer model. Magn Reson Med 2003;49:398-401.

28. Ewing JR, Cao Y, Fenstermacher J. Single-coil arterial spintagging for estimating cerebral blood flow as viewed from the capillary: relative contributions of intra- and extravascular signal. Magn Reson Med 2001;46:465-475.

29. Parkes LM, Tofts PS. Improved accuracy of human cerebral blood perfusion measurements using arterial spin labeling: accounting for capillary water permeability. Magn Reson Med 2002;48: 27-41.

30. Detre JA, Leigh JS, Williams DS, Koretsky AP. Perfusion imaging. Magn Reson Med 1992;23:37-45.

31. Williams DS, Detre JA, Leigh JS, Koretsky AP. Magnetic resonance imaging of perfusion using spin inversion of arterial water. Proc Natl Acad Sci U S A 1992;89:212-216.

32. Edelman RR, Siewert B, Darby DG, et al. Qualitative mapping of cerebral blood flow and functional localization with echo-planar MR imaging and signal targeting with alternating radio frequency. Radiology 1994;192:513-520.

33. Kim SG. Quantification of relative cerebral blood flow change by flow-sensitive alternating inversion recovery (FAIR) technique: application to functional mapping. Magn Reson Med 1995;34: 293-301.

34. Silva AC, Zhang W, Williams DS, Koretsky AP. Multislice MRI of rat brain perfusion during amphetamine stimulation using arterial spin labeling. Magn Reson Med 1995;33:209-214.
35. Zaharchuk G, Ledden PJ, Kwong KK, Reese TG, Rosen BR, Wald LL. Multislice perfusion and perfusion territory imaging in humans with separate label and image coils. Magn Reson Med 1999;41:1093-1098.

36. Zhang W, Silva AC, Williams DS, Koretsky AP. NMR measurement of perfusion using arterial spin labeling without saturation of macromolecular spins. Magn Reson Med 1995;33:370-376.

37. Kao YH, Wan X, MacFall JR. Simultaneous multislice acquisition with arterial-flow tagging (SMART) using echo planar imaging (EPI). Magn Reson Med 1998;39:662-665.

38. Yang Y, Frank JA, Hou L, Ye FQ, McLaughlin AC, Duyn JH Multislice imaging of quantitative cerebral perfusion with pulsed arterial spin labeling. Magn Reson Med 1998;39:825-832.

39. Yongbi MN, Fera F, Yang Y, Frank JA, Duyn JH. Pulsed arterial spin labeling: comparison of multisection baseline and functional MR imaging perfusion signal at 1.5 and $3.0 \mathrm{~T}$ : initial results in six subjects. Radiology 2002;222:569-575.

40. Wang J, Zhang Y, Wolf RL, Roc AC, Alsop DC, Detre JA. Amplitude-modulated continuous arterial spin-labeling 3.0-T perfusion MR imaging with a single coil: feasibility study. Radiology 2005;235:218-228.

41. Wang Z, Wang J, Connick TJ, Wetmore GS, Detre JA. Continuous ASL (CASL) perfusion MRI with an array coil and parallel imaging at 3T. Magn Reson Med 2005;54:732-737.

42. Garcia DM, de Bazelaire C, Alsop DC. Pseudo-continuous flow driven adiabatic inversion for arterial spin labeling [abstract] Proc Int Soc Magn Reson Med 2005:37.

43. Fernández-Seara MA, Wang Z, Wang J, et al. Continuous arterial spin labeling perfusion measurements using single shot 3D GRASE at 3 T. Magn Reson Med 2005;54:1241-1247.

44. Ye FQ, Frank JA, Weinberger DR, McLaughlin AC. Noise reduction in 3D perfusion imaging by attenuating the static signal in arterial spin tagging (ASSIST). Magn Reson Med 2000;44:92100.

45. Fernández-Seara MA, Wang J, Wang Z, et al. Imaging mesial temporal lobe activation during scene encoding: comparison of fMRI using BOLD and ASL. Hum Brain Mapp May 24, 2007 [Epub ahead of print].

46. Siewert B, Schlaug G, Edelman RR, Warach S. Comparison of EPISTAR and T2*-weighted gadolinium-enhanced perfusion imaging in patients with acute cerebral ischemia. Neurology 1997 48:673-679.

47. Detre JA, Alsop DC, Vives LR, Maccotta L, Teener JW, Raps EC. Noninvasive MRI evaluation of cerebral blood flow in cerebrovascular disease. Neurology 1998;50:633-641.

48. Chalela JA, Alsop DC, Gonzalez-Atavales JB, Maldjian JA, Kasner SE, Detre JA. Magnetic resonance perfusion imaging in acute ischemic stroke using continuous arterial spin labeling. Stroke 2000;31:680-687.

49. Wolf RL, Alsop DC, McGarvey ML, Maldjian JA, Wang J, Detre JA. Susceptibility contrast and arterial spin labeled perfusion MRI in cerebrovascular disease. J Neuroimaging 2003;13:17-27.

50. Hunsche S, Sauner D, Schreiber WG, Oelkers P, Stoeter P. FAIR and dynamic susceptibility contrast-enhanced perfusion imaging in healthy subjects and stroke patients. J Magn Reson Imaging 2002;16:137-146.

51. Yoneda K, Harada M, Morita N, Nishitani H, Uno M, Matsuda T. Comparison of FAIR technique with different inversion times and post contrast dynamic perfusion MRI in chronic occlusive cerebrovascular disease. Magn Reson Imaging 2003;21:701-705.

52. Gunther M, Bock M, Schad LR. Arterial spin labeling in combination with a look-locker sampling strategy: inflow turbo-sampling EPI-FAIR (ITS-FAIR). Magn Reson Med 2001;46:974984.

53. Hendrikse J, van Osch MJ, Rutgers DR, et al. Internal carotid artery occlusion assessed at pulsed arterial spin-labeling perfusion MR imaging at multiple delay times. Radiology 2004;233:899904.

54. Petersen ET, Lim T, Golay X. Model-free arterial spin labeling quantification approach for perfusion MRI. Magn Reson Med 2006;55:219-232. 
55. Duhamel G, de Bazelaire C, Alsop DC. Evaluation of systematic quantification errors in velocity-selective arterial spin labeling of the brain. Magn Reson Med 2003;50:145-153.

56. Wong EC. Quantifying CBF with pulsed ASL: technical and pulse sequence factors. J Magn Reson Imaging 2005;22:727-731.

57. Wong EC, Cronin M, Wu WC, Inglis B, Frank LR, Liu TT. Velocity-selective arterial spin labeling. Magn Reson Med 2006; 55:1334-1341.

58. Wang J, Alsop DC, Song HK, et al. Arterial transit time imaging with flow encoding arterial spin tagging (FEAST). Magn Reson Med 2003;50:599-607.

59. Detre JA, Samuels OB, Alsop DC, Gonzalez-At JB, Kasner SE, Raps EC. Noninvasive magnetic resonance imaging evaluation of cerebral blood flow with acetazolamide challenge in patients with cerebrovascular stenosis. J Magn Reson Imaging 1999;10:870875 .

60. Ances BM, McGarvey ML, Abrahams JM, et al. Continuous arterial spin labeled perfusion magnetic resonance imaging in patients before and after carotid endarterectomy. J Neuroimaging 2004;14:133-138

61. Floyd TF, Ratcliffe SJ, Wang J, Resch B, Detre JA. Precision of the CASL-perfusion MRI technique for the measurement of cerebral blood flow in whole brain and vascular territories. J Magn Reson Imaging 2003;18:649-655.

62. Jahng GH, Song E, Zhu XP, Matson GB, Weiner MW, Schuff N. Human brain: reliability and reproducibility of pulsed arterial spin-labeling perfusion MR imaging. Radiology 2005;234:909916.

63. Parkes LM, Rashid W, Chard DT, Tofts PS. Normal cerebral perfusion measurements using arterial spin labeling: reproducibility, stability, and age and gender effects. Magn Reson Med 2004; 51:736-743.

64. Yen YF, Field AS, Martin EM, et al. Test-retest reproducibility of quantitative CBF measurements using FAIR perfusion MRI and acetazolamide challenge. Magn Reson Med 2002;47:921-928.

65. Kimura H, Kado H, Koshimoto Y, Tsuchida T, Yonekura Y, Itoh H. Multislice continuous arterial spin-labeled perfusion MRI in patients with chronic occlusive cerebrovascular disease: a correlative study with $\mathrm{CO}_{2}$ PET validation. J Magn Reson Imaging 2005;22:189-198.

66. Jefferson AL, Glosser G, Detre JA, Sinson G, Liebeskind DS Neuropsychological and perfusion MR imaging correlates of revascularization in a case of moyamoya syndrome. AJNR Am J Neuroradiol 2006;27:98-100.

67. Last D, Alsop DC, Abduljalil AM, et al. Global and regional effects of type 2 diabetes mellitus on brain tissue volumes and cerebral vasoreactivity. Diabetes Care 2007;30:1193-1199 [Epub ahead of print].

68. van Laar PJ, van Raamt AF, van der Grond J, Mali WPTM, van der Graaf Y, Hendrikse J; SMART Study Group. Increasing levels of TNF $\alpha$ are associated with increased brain perfusion. Atherosclerosis Jan 11, 2007 [Epub ahead of print].

69. Gunther M. Efficient visualization of vascular territories in the human brain by cycled arterial spin labeling MRI. Magn Reson Med 2006;56:671-675.

70. Jones CE, Wolf RL, Detre JA, et al. Structural MRI of carotid artery atherosclerotic lesion burden and characterization of hemispheric cerebral blood flow before and after carotid endarterectomy. NMR Biomed 2006;19:198-208.

71. Werner R, Alfke K, Schaeffter T, Nabavi A, Mehdorn HM, Jansen O. Brain perfusion territory imaging applying obliqueplane arterial spin labeling with a standard send/receive head coil. Magn Reson Med 2004;52:1443-1447.

72. Davies NP, Jezzard P. Selective arterial spin labeling (SASL): perfusion territory mapping of selected feeding arteries tagged using two-dimensional radiofrequency pulses. Magn Reson Med 2003;49:1133-1142.

73. Werner R, Norris DG, Alfke K, Mehdorn HM, Jansen O. Continuous artery-selective spin labeling (CASSL). Magn Reson Med 2005;53:1006-1012.

74. Hendrikse J, van der Zwan A, Ramos LM, et al. Altered flow territories after extracranial-intracranial bypass surgery. Neurosurgery 2005;57:486-494; discussion 486-494.
75. van Laar PJ, Hendrikse J, Golay X, Lu H, van Osch MJ, van der Grond J. In vivo flow territory mapping of major brain feeding arteries. Neuroimage 2006;29:136-144.

76. Dixon WT, Du LN, Faul DD, Gado M, Rossnick S. Projection angiograms of blood labeled by adiabatic fast passage. Magn Reson Med 1986;3:454-462.

77. Nishimura DG, Macovski A, Pauly JM. Considerations of magnetic resonance angiography by selective inversion recovery. Magn Reson Med 1988;7:472-484.

78. Nishimura DG, Macovski A, Pauly JM, Conolly SM. MR angiography by selective inversion recovery. Magn Reson Med 1987;4:193-202.

79. Wolf RL, Wang J, Detre JA, Zager EL, Hurst RW. AV shunt visualization with arterial spin labeled perfusion MR imaging [abstract]. Proc Int Soc Magn Reson Med 2006:2697.

80. Wolf RL, Wang J, Wang S, et al. Grading of CNS neoplasms using continuous arterial spin labeled perfusion MR imaging at 3 tesla. J Magn Reson Imaging 2005;22:475-482.

81. Wang J, Fernández-Seara MA, Wang S, St Lawrence KS. When perfusion meets diffusion: in vivo measurement of water permeability in human brain. J Cereb Blood Flow Metab 2007;27:839849.

82. Warmuth C, Gunther M, Zimmer C. Quantification of blood flow in brain tumors: comparison of arterial spin labeling and dynamic susceptibility-weighted contrast-enhanced MR imaging. Radiology 2003;228:523-532.

83. Gaa J, Warach S, Wen P, Thangaraj V, Wielopolski P, Edelman RR. Noninvasive perfusion imaging of human brain tumors with EPISTAR. Eur Radiol 1996;6:518-522.

84. Kimura H, Takeuchi H, Koshimoto Y, et al. Perfusion imaging of meningioma by using continuous arterial spin-labeling: comparison with dynamic susceptibility-weighted contrast-enhanced MR images and histopathologic features. AJNR Am J Neuroradiol 2006;27:85-93.

85. Weber MA, Zoubaa S, Schlieter M, et al. Diagnostic performance of spectroscopic and perfusion MRI for distinction of brain tumors. Neurology 2006;66:1899-1906.

86. Law M, Yang S, Wang H, et al. Glioma grading: sensitivity, specificity, and predictive values of perfusion MR imaging and proton MR spectroscopic imaging compared with conventional MR imaging. AJNR Am J Neuroradiol 2003;24:1989-1998.

87. Weber MA, Thilmann C, Lichy MP, et al. Assessment of irradiated brain metastases by means of arterial spin-labeling and dynamic susceptibility-weighted contrast-enhanced perfusion MRI: initial results. Invest Radiol 2004;39:277-287.

88. Cha S, Knopp EA, Johnson G, et al. Dynamic contrast-enhanced T2-weighted MR imaging of recurrent malignant gliomas treated with thalidomide and carboplatin. AJNR Am J Neuroradiol 2000; 21:881-890.

89. Law M, Oh S, Babb JS, et al. Low-grade gliomas: dynamic susceptibility-weighted contrast-enhanced perfusion MR imaging--prediction of patient clinical response. Radiology 2006;238: $658-667$.

90. Law M, Oh S, Johnson G, et al. Perfusion magnetic resonance imaging predicts patient outcome as an adjunct to histopathology: a second reference standard in the surgical and nonsurgical treatment of low-grade gliomas. Neurosurgery 2006;58:1099-1107; discussion 1099-1107.

91. Fink GR, Pawlik G, Stefan H, Pietrzyk U, Wienhard K, Heiss WD. Temporal lobe epilepsy: evidence for interictal uncoupling of blood flow and glucose metabolism in temporomesial structures. J Neurol Sci 1996;137:28-34.

92. Gaillard WD, Fazilat S, White S, et al. Interictal metabolism and blood flow are uncoupled in temporal lobe cortex of patients with complex partial epilepsy. Neurology 1995;45:1841-1847.

93. Leiderman DB, Balish M, Sato S, et al. Comparison of PET measurements of cerebral blood flow and glucose metabolism for the localization of human epileptic foci. Epilepsy Res 1992;13: 153-157.

94. Detre JA, Alsop DC. Perfusion magnetic resonance imaging with continuous arterial spin labeling: methods and clinical applications in the central nervous system. Eur J Radiol 1999;30:115124. 
95. Wolf RL, Alsop DC, Levy-Reis I, et al. Detection of mesial temporal lobe hypoperfusion in patients with temporal lobe epilepsy by use of arterial spin labeled perfusion MR imaging. AJNR Am J Neuroradiol 2001;22:1334-1341.

96. Wu RH, Bruening R, Noachtar S, et al. MR measurement of regional relative cerebral blood volume in epilepsy. J Magn Reson Imaging 1999;9:435-440.

97. Liu HL, Kochunov P, Hou J, et al. Perfusion-weighted imaging of interictal hypoperfusion in temporal lobe epilepsy using FAIRHASTE: comparison with $\mathrm{H}_{2}{ }^{15} \mathrm{O}$ PET measurements. Magn Reson Med 2001;45:431-435.

98. Alsop DC, Detre JA. Background suppressed 3D RARE arterial spin labeled perfusion MRI [abstract]. Proc Int Soc Magn Reson Med 1999:601.

99. Crelier GR, Hoge RD, Munger P, Pike GB. Perfusion-based functional magnetic resonance imaging with single-shot RARE and GRASE acquisitions. Magn Reson Med 1999;41:132-136.

100. Wang J, Li L, Roc AC, et al. Reduced susceptibility effects in perfusion fMRI with single-shot spin-echo EPI acquisitions at 1.5 tesla. Magn Reson Imaging 2004;22:1-7.

101. Stefanovic B, Warnking JM, Kobayashi E, et al. Hemodynamic and metabolic responses to activation, deactivation and epileptic discharges. Neuroimage 2005;28:205-215.

102. Wang J, Licht DJ. Pediatric perfusion MR imaging using arterial spin labeling. Neuroimaging Clin N Am 2006;16:149-167, ix.

103. Wang J, Licht DJ, Jahng GH, et al. Pediatric perfusion imaging using pulsed arterial spin labeling. J Magn Reson Imaging 2003; 18:404-413.

104. Floyd TF, McGarvey M, Ochroch EA, et al. Perioperative changes in cerebral blood flow after cardiac surgery: influence of anemia and aging. Ann Thorac Surg 2003;76:2037-2042.

105. Alsop DC, Fearing MA, Johnson K, Sperling R, Fong TG, Inouye SK. The role of neuroimaging in elucidating delirium pathophysiology. J Gerontol A Biol Sci Med Sci 2006;61:1287-1293.

106. Sandson TA, O'Connor M, Sperling RA, Edelman RR, Warach S. Noninvasive perfusion MRI in Alzheimer's disease: a preliminary report. Neurology 1996;47:1339-1342.

107. Alsop DC, Detre JA, Grossman M. Assessment of cerebral blood flow in Alzheimer's disease by spin-labeled magnetic resonance imaging. Ann Neurol 2000;47:93-100.

108. Johnson NA, Jahng GH, Weiner MW, et al. Pattern of cerebral hypoperfusion in Alzheimer disease and mild cognitive impairment measured with arterial spin-labeling MR imaging: initial experience. Radiology 2005;234:851-859.
109. Du AT, Jahng GH, Hayasaka S, et al. Hypoperfusion in frontotemporal dementia and Alzheimer disease by arterial spin labeling MRI. Neurology 2006;67:1215-1220.

110. Fong T, Press D, Alsop DC. Greater blood flow reduction in mild diffuse Lewy body disease than in mild Alzheimer's disease [abstract]. Proc Int Soc Magn Reson Med 2006:719.

111. Licht DJ, Wang J, Silvestre DW, et al. Preoperative cerebral blood flow is diminished in neonates with severe congenital heart defects. J Thorac Cardiovasc Surg 2004;128:841-849.

112. Oguz KK, Golay X, Pizzini FB, et al. Sickle cell disease: continuous arterial spin-labeling perfusion MR imaging in children. Radiology 2003;227:567-574.

113. Strouse JJ, Cox CS, Melhem ER, et al. Inverse correlation between cerebral blood flow measured by continuous arterial spinlabeling (CASL) MRI and neurocognitive function in children with sickle cell anemia (SCA). Blood 2006;108:379-381.

114. Zarahn E, Aguirre GK, D'Esposito M. Empirical analyses of BOLD fMRI statistics. I. Spatially unsmoothed data collected under null-hypothesis conditions. Neuroimage 1997;5:179-197.

115. Aguirre GK, Detre JA, Zarahn E, Alsop DC. Experimental design and the relative sensitivity of BOLD and perfusion fMRI. Neuroimage 2002;15:488-500.

116. Wang J, Aguirre GK, Kimberg DY, Roc AC, Li L, Detre JA. Arterial spin labeling perfusion fMRI with very low task frequency. Magn Reson Med 2003;49:796-802.

117. Olson IR, Rao H, Moore KS, Wang J, Detre JA, Aguirre GK. Using perfusion fMRI to measure continuous changes in neural activity with learning. Brain Cogn 2006;60:262-271.

118. O'Gorman RL, Kumari V, Williams SC, et al. Personality factors correlate with regional cerebral perfusion. Neuroimage 2006;31: 489-495.

119. Rao H, Gillihan SJ, Wang J, et al. Genetic variation in serotonin transporter alters resting brain function in healthy individuals. Biol Psychiatry 2007 May 2 [Epub ahead of print].

120. Kim J, Whyte J, Wang J, Rao H, Tang KZ, Detre JA. Continuous ASL perfusion fMRI investigation of higher cognition: quantification of tonic CBF changes during sustained attention and working memory tasks. Neuroimage 2006;31:376-385.

121. Rao H, Wang J, Tang K, Pan W, Detre JA. Imaging brain activity during natural vision using CASL perfusion fMRI. Hum Brain Mapp 2006 Oct. 10 [Epub ahead of print].

122. Wang J, Rao H, Wetmore GS, et al. Perfusion functional MRI reveals cerebral blood flow pattern under psychological stress. Proc Natl Acad Sci U S A 2005;102:17804-17809. 